\title{
Immunohistochemical analysis of claudin expression in pancreatic cystic tumors
}

\author{
JIN HA LEE ${ }^{1}$, KYUNG SIK KIM², TAE-JUNG KIM ${ }^{3}$, SUNG PIL HONG ${ }^{1}$, \\ SI YOUNG SONG ${ }^{1}$, JAE BOCK CHUNG ${ }^{1}$ and SEUNG WOO PARK ${ }^{1}$
}

\begin{abstract}
Departments of ${ }^{1}$ Internal Medicine and Institute of Gastroenterology, and ${ }^{2}$ Surgery, Yonsei University College of Medicine, Seoul 120-752; ${ }^{3}$ Department of Hospital Pathology, The Catholic University of Korea, Seoul 137-701, Republic of Korea
\end{abstract}

Received September 9, 2010; Accepted December 3, 2010

DOI: $10.3892 /$ or.2011.1132

\begin{abstract}
Aberrant expression of the claudin family of proteins has been reported in many human cancers, including pancreatic ductal carcinoma. Intraductal papillary mucinous neoplasms of the pancreas (IPMN) and mucinous cystic neoplasms (MCN) are considered precancerous lesions that are able to progress towards pancreatic ductal adenocarcinoma. We analyzed the expression of several claudin family members using surgical IPMN and MCN specimens to clarify the relationships between claudin expression and clinicopathological features. Twenty-nine and 25 consecutive cases of IPMN and MCN were selected and the expression of claudin-2, -4 and -18 was analyzed by immunohistochemistry. In addition, IPMN and MCN histological grade as well as IPMN subtypes were analyzed in relation to claudin expression. The 29 cases of IPMN comprised of $3(10.3 \%)$ adenomas, $18(62.1 \%)$ borderline malignancies and 8 $(27.6 \%)$ carcinomas. The 25 cases of MCN comprised of 13 $(52 \%)$ adenomas, $5(20 \%)$ borderline malignancies and 7 (28\%) carcinomas. Claudin-2, -4 and -18 showed strong expression both in IPMN and MCN, with the exception of claudin-4 in MCN. The expression grades of claudin-2 in both IPMN and MCN became weaker with increased histological grade. On the other hand, the expression grades of claudin-4 and -18 became stronger with increased histological grade in both IPMN and MCN. With regard to histological subtype, claudin-4 expression was the strongest in pancreatobiliary type IPMN, and claudin-18 expression was the strongest in gastric type IPMN. The distinct expression patterns of claudin-2, -4 and -18 suggest that claudins may
\end{abstract}

Correspondence to: Dr Seung Woo Park, Department of Internal Medicine and Institute of Gastroenterology, Yonsei University College of Medicine, 250 Seongsanno, Seodaemun-gu, Seoul 120-752, Republic of Korea

E-mail: swoopark@yuhs.ac

Key words: claudins, immunohistochemistry, intraductal papillary mucinous neoplasms, mucinous cystic neoplasms, pancreas serve as useful molecular markers for tumor differentiation and progression in IPMN and MCN.

\section{Introduction}

Recent advances in diagnostic imaging have resulted in more frequent detection of small pancreatic cystic lesions, such as serous cystic tumors, intraductal papillary mucinous neoplasms (IPMN), mucinous cystic neoplasms (MCN), and solid pseudo-papillary neoplasms $(1,2)$. Of these pancreatic cystic lesions, mucinous tumors such as IPMN and MCN are considered definite premalignant lesions (3). Because they show a broad spectrum of dysplasia, ranging from adenoma and borderline neoplasm to carcinoma, the identifying factors associated with the progression of non-invasive and/or invasive carcinoma is important $(4,5)$. IPMN and MCN of the pancreas have similar histopathological characteristics. Nevertheless, they are clinically and epidemiologically quite different, and the relationships between them have long been a matter of discussion $(2,6,7)$.

Tight junctions are intercellular adhesion structures forming a permeability barrier at the apical surfaces of epithelia, and giving polarity to these cells (8-11). The claudin family of proteins is an essential component of the tight junction, consisting at least 23 transmembrane proteins (8). The loss of claudins and other tight junction proteins in cancer has been interpreted as a mechanism for the loss of cell adhesion and as an important step in the progression of cancer to metastasis $(12,13)$. Previous studies have shown that claudins are down-regulated or up-regulated in various cancers (14-21).

Microarray analysis (unpublished data) performed in our laboratory revealed differential expression of various tight junction proteins in IPMN and normal tissue. Among the tight junction proteins, claudin-2, 4 , and -18 were significantly up-regulated in IPMN compared to normal tissue. This raised question about the differential expression patterns of those proteins in IPMN, MCN, and normal pancreas. The aims of this study were to analyze the expression patterns of claudin-2, -4 and -18 in mucinous tumors of the pancreas using surgical IPMN and MCN specimens to clarify the relationships between claudin expression and clinicopathological features such as histological grade and histological subtype. 
Table I. Clinicopathological findings of patients with intraductal papillary mucinous neoplasm (IPMN) and mucinous cystic neoplasm (MCN) of the pancreas.

\begin{tabular}{|c|c|c|c|}
\hline & $\begin{array}{c}\text { IPMN } \\
(n=29)(\%)\end{array}$ & $\begin{array}{c}\text { MCN } \\
(n=25)(\%)\end{array}$ & $\mathrm{P}$-value \\
\hline $\begin{array}{l}\text { Age } \\
\qquad(\text { years, mean } \pm \text { SD) }\end{array}$ & $61.9 \pm 6.8$ & $49 \pm 12.3$ & $<0.001$ \\
\hline $\begin{array}{l}\text { Sex } \\
\text { Male } \\
\text { Female }\end{array}$ & $\begin{array}{l}19(65.5) \\
10(34.5)\end{array}$ & $\begin{array}{r}3(12.5) \\
21(87.5)\end{array}$ & $<0.001$ \\
\hline $\begin{array}{l}\text { Site } \\
\text { Head } \\
\text { Body/tail }\end{array}$ & $\begin{array}{r}22(75.9) \\
7(24.1)\end{array}$ & $\begin{array}{c}2(8.3) \\
22(91.7)\end{array}$ & $<0.001$ \\
\hline $\begin{array}{l}\text { Tumor size } \\
\qquad(\mathrm{mm}, \text { mean } \pm \mathrm{SD})\end{array}$ & $31.6 \pm 11.3$ & $54.6 \pm 36.9$ & $<0.001$ \\
\hline $\begin{array}{l}\text { Histological grade } \\
\text { (WHO classification) }\end{array}$ & & & 0.001 \\
\hline $\begin{array}{l}\text { Adenoma } \\
\text { Borderline } \\
\text { Carcinoma } \\
\text { Non-invasive } \\
\text { Invasive }\end{array}$ & $\begin{aligned} 3 & (10.3) \\
18 & (62.1) \\
8 & (27.6) \\
4 & (13.8) \\
4 & (13.8)\end{aligned}$ & $\begin{aligned} 13 & (52) \\
5 & (20) \\
7 & (28) \\
6 & (24) \\
1 & (4)\end{aligned}$ & \\
\hline $\begin{array}{l}\text { Histological subtype } \\
\text { Gastric type } \\
\text { Intestinal type } \\
\text { Pancreato-biliary type }\end{array}$ & $\begin{array}{r}11(37.9) \\
13(44.8) \\
5(17.2)\end{array}$ & & \\
\hline
\end{tabular}

\section{Materials and methods}

Patients and tissue specimens. Consecutive cases of IPMN $(n=29)$ and $\operatorname{MCN}(n=25)$ were selected from the surgical pathology files at Severance Hospital, Yonsei University, Seoul, Korea, between January 1995 and December 2007. One sample of normal pancreatic tissue and one sample of chronic pancreatitis tissue were obtained for control analysis. Tumors were histologically graded and staged according to the World Health Organization (WHO) tumor classification system (5), and the histological subtypes of IPMN were classified as: gastric, intestinal, pancreatobiliary, and oncocytic (22). The subtypes were based on their histological phenotypes and immunohistochemical expression of MUC proteins (gastric type, MUC1-/MUC2-; intestinal type, MUC1-/MUC2+; pancreato-biliary type, $\mathrm{MUC1}^{+} / \mathrm{MUC2}^{-}$; and oncocytic type, $\mathrm{MUC1}^{+} / \mathrm{MUC2}^{-}$). Formalin-fixed, paraffin-embedded tissues were used for immunohistochemistry.

Immunohistochemistry. The primary antibodies were polyclonal rabbit anti-claudin-2 (Abcam Inc., Cambridge, MA, USA; 1:200 dilution), polyclonal goat anti-claudin-4 (Santa Cruz Biotechnology, Santa Cruz, CA, USA; 1:200 dilution), and polyclonal goat anti-claudin-18 (Santa Cruz Biotechnology; 1:200 dilution). Sections (4 $\mu \mathrm{m})$ from formalin-fixed, paraffin-embedded tissues were deparaffinized with xylene, then rehydrated with graded alcohol. Endogenous peroxidase activity was blocked by incubating slides at room temperature for $15 \mathrm{~min}$ in methanol containing $3 \%$ hydrogen peroxide. Microwave antigen retrieval was performed in citrate buffer $(0.01 \mathrm{M}, \mathrm{pH} 6.0)$ for $15 \mathrm{~min}$. To reduce non-specific staining, slides were incubated in a $10 \%$ normal goat serum solution for $60 \mathrm{~min}$. Blocked sections were incubated in primary antibody overnight at $4^{\circ} \mathrm{C}$ and stained with a streptavidin-biotin-peroxidase kit according to the manufacturer's protocol. Slides were developed by incubating with 3-amino-9-ethyl carbazole and were counterstained with haematoxylin.

Evaluation of immunohistochemical staining of claudin. The result of immunohistochemical staining was scored on the basis of intensity and extent of staining ( 0 , no staining; 1 , weak membranous staining and/or $<50 \%$ staining; 2 , strong membranous staining and $\geq 50 \%$ staining). Because IPMN and MCN often have variable epithelial dysplasia within the same tumor, the intensity of immunohistochemical claudin staining was evaluated in the region in each neoplasm with the highest degree of dysplasia. Cases with a score of 0 were interpreted as negative. Cases scored as 1 or 2 were interpreted as positive for claudin expression. The evaluation was performed independently by two investigators (J.H.L. and T.J.K) without any prior knowledge of the patients' clinical information.

Statistical analysis. SPSS ${ }^{\circledR}$ version 15.0 (SPSS Inc., Chicago, IL, USA) was used for statistical analysis, and a value of $\mathrm{p}<0.05$ was considered significant. Continuous data were analyzed using Student's t-test, and categorical data were compared by Pearson's $\chi^{2}$ test or Fisher's exact test. Fisher's exact test was used to assess the association between the histological features and claudin IHC.

\section{Results}

Clinicopathological findings. Clinicopathological findings from 29 cases of IPMN and 25 cases of MCN are summarized in Table I. Among the 29 cases of IPMN, there were $2(10.3 \%)$ adenomas, $18(62.1 \%)$ borderline malignancies, and $8(27.6 \%)$ carcinomas. Of the 29 cases of IPMN, 18 (62.1\%) were gastric type, $9(31 \%)$ were intestinal type, and $2(6.9 \%)$ were pancreato-biliary type. Among the 25 cases of $\mathrm{MCN}$, there were $13(52 \%)$ adenomas, $5(20 \%)$ borderline malignancies, and $7(28 \%)$ carcinomas. MCN patients were predominantly female $(87.5 \%, \mathrm{p}<0.001)$ and were significantly younger $(49 \pm 12.3$ years vs. $61.9 \pm 6.8$ years, $p<0.001)$ than IPMN patients. MCNs were exclusively located in the body and tail of the pancreas, while IPMN localized primarily to the head $(75.9 \%, \mathrm{p}<0.001)$. MCN masses were significantly larger than IPMN masses $(54.6 \pm 36.9$ vs. $31.6 \pm 11.3 \mathrm{~mm}$, $\mathrm{P}<0.001)$.

Immunohistochemical findings. In normal human pancreatic tissue (Fig. 1), claudin-2 showed weak membrane-bound, cytoplasmic granular staining in acinar cells, ductal cells, and islet cells. Claudin- 4 showed strong membranous staining in 

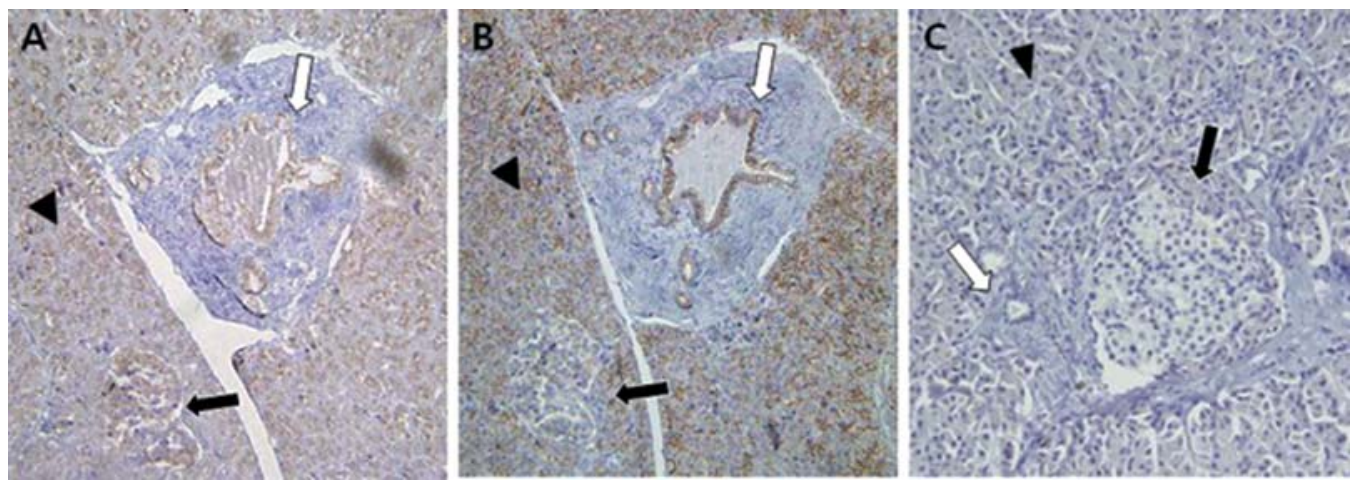

Figure 1. Claudin expression in normal pancreatic tissue. (A) Claudin-2 shows weak membranous granular staining in acinar cells (black arrow head), ductal cells (white arrow) and islet cells (black arrow). (B) Claudin-4 shows strong membranous staining in acinar cells and ductal cells but not in islet cells. (C) Claudin-18 shows negative staining in ductal epithelium, acini, and islets of Langerhans. (A-C, x400).

Table II. Claudin-2, -4 , and -18 expression according to histological grade of IPMN.

\begin{tabular}{lccc}
\hline & $\begin{array}{c}\text { Adenoma } \\
(\mathrm{n}=3)\end{array}$ & $\begin{array}{c}\text { Borderline } \\
(\mathrm{n}=18)\end{array}$ & $\begin{array}{c}\text { Carcinoma } \\
(\mathrm{n}=8)\end{array}$ \\
\hline Claudin-2 & & & \\
Negative & 0 & 3 & 5 \\
+ & 0 & 7 & 0 \\
++ & 3 & 8 & 3 \\
Claudin-4 & & & \\
$\quad$ Negative & 3 & 2 & 1 \\
+ & 0 & 12 & 1 \\
++ & 0 & 4 & 6 \\
Claudin-18 & & & \\
$\quad$ Negative & 1 & 0 & 0 \\
+ & 1 & 11 & 1 \\
++ & 1 & 7 & 7 \\
\hline
\end{tabular}

IPMN, intraductal papillary mucinous neoplasm.

acinar cells and ductal cells but not in islet cells. Claudin-18 staining was negative in ductal epithelium, acinar cells, or in islet cells

The comparison of claudin immunohistochemistry with histological grade is summarized in Tables II and III. IPMNs were positive for claudin-2, -4 and -18 in $72.4,89.7$ and $96.6 \%$ of cases, respectively. MCNs were positive for clauding-2, -4 and -18 in $60.0,44.0$ and $96.0 \%$ of cases, respectively. There was a tendency for higher expression of all claudins tested in IPMN than in MCN. The expression of claudin-2 became weaker as the histological grade increased both in IPMN and MCN (IPMN, p=0.025; MCN, p=0.025). All 3 IPMN adenomas (100\%) revealed strong claudin-2 expression, whereas 5 out of 8 IPMN carcinomas $(62.5 \%)$ revealed negative expression for claudin-2 (Fig. 2A-C). For MCN, 8 out of $13(61.5 \%)$ adenomas showed strong claudin-2 expression, whereas 6 out of $7(85.7 \%)$ carcinomas showed negative for claudin-2 expression (Fig. 3A-C). Contrary to claudin-2 expression, claudin-4 showed more robust expression
Table III. Claudin-2, -4 , and -18 expression according to histological grade of MCN.

\begin{tabular}{lccc}
\hline & $\begin{array}{c}\text { Adenoma } \\
(\mathrm{n}=13)\end{array}$ & $\begin{array}{c}\text { Borderline } \\
(\mathrm{n}=5)\end{array}$ & $\begin{array}{c}\text { Carcinoma } \\
(\mathrm{n}=7)\end{array}$ \\
\hline Claudin-2 & & & \\
$\quad$ Negative & 3 & 1 & 6 \\
+ & 2 & 1 & 1 \\
++ & 8 & 3 & 0 \\
Claudin-4 & & & \\
$\quad$ Negative & 11 & 0 & 3 \\
+ & 1 & 5 & 2 \\
++ & 1 & 0 & 2 \\
Claudin-18 & & & \\
Negative & 1 & 0 & 0 \\
+ & 7 & 1 & 0 \\
++ & 5 & 4 & 7 \\
\hline
\end{tabular}

MCN, mucinous cystic neoplasm.

as the histological grade increased both in IPMN and MCN (IPMN, $\mathrm{p}=0.001 ; \mathrm{MCN}, \mathrm{p}=0.001$ ). While all 3 IPMN adenomas (100\%) were negative for claudin-4, 6 out of 8 (75.0\%) IPMN carcinomas were strongly positive for claudin- 4 expression (Fig. 2D-F). In MCN, 11 of 13 (84.6\%) adenomas were negative for claudin-4, whereas 2 of 7 (28.6\%) carcinomas showed strong claudin-4 expression (Fig. 3D-F). The expression patterns of claudin-18 were similar to those of claudin-4 (IPMN, p=0.015; MCN, p=0.030). One of the 3 $(33.3 \%)$ IPMN adenomas showed negative expression for claudin-18, whereas 7 of $8(87.5 \%)$ IPMN carcinomas were strong positive for claudin-18 expression (Fig. 2G-I). In MCN, 8 adenomas $(61.5 \%)$ showed negative or weak for claudin-18 expression, whereas all 7 carcinomas (100\%) showed strong claudin-18 expression (Fig. 3G-I).

In some cases of IPMN and MCN which differ in grade of dysplasia in the same section, more advanced area, i.e. carcinoma revealed stronger expression for claudin- 4 or -18 


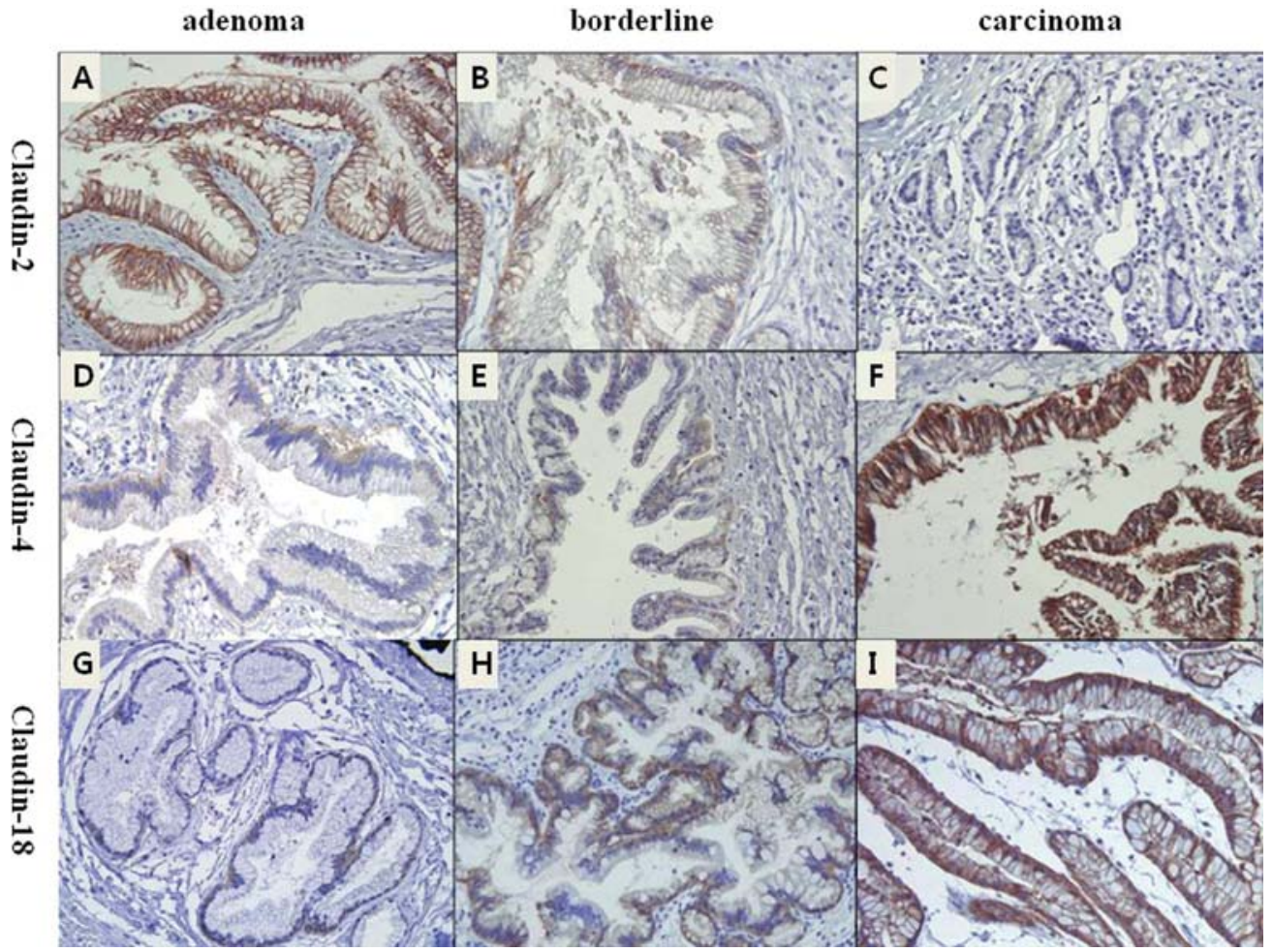

Figure 2. Claudin-2, -4 , and -18 expression in IPMN by immunochemistry. (A) Strong intensity claudin-2 staining in IPMN adenomas (score 2). (B) Weak intensity claudin-2 staining in borderline IPMN (score 1). (C) Negative claudin-2 staining in IPMN carcinoma (score 0). (D) Negative claudin-4 staining in IPMN adenomas (score 0). (E) Weak intensity claudin-4 staining in a borderline IPMN (score 1). (F) Strong intensity claudin-4 staining in an IPMN carcinoma (score 2). (G) Negative claudin-18 staining in an IPMN adenoma (score 0). (H) Weak intensity claudin-18 staining in a borderline IPMN (score 1). (I) Strong intensity claudin-18 staining in an IPMN carcinoma (score 2) (A-I, x400).

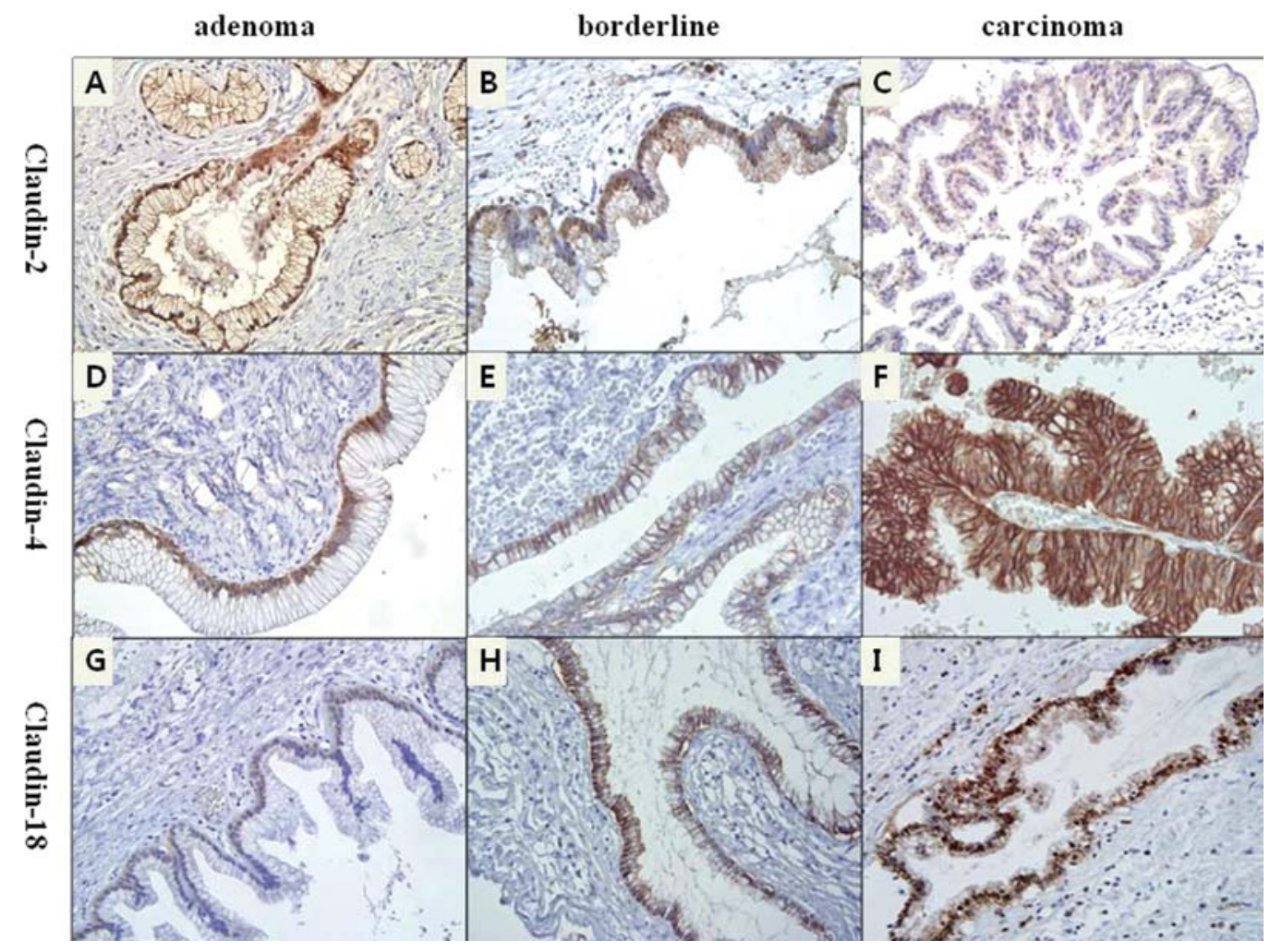

Figure 3. Claudin-2, -4, and -18 expression in MCN by immunochemistry. (A) Strong intensity claudin-2 staining in an MCN adenoma (score 2). (B) Weak intensity claudin-2 staining in a borderline MCN (score 1). (C) Negative claudin-2 staining in a MCN carcinoma (score 0). (D) Negative claudin-4 staining in an MCN adenoma (score 0). (E) Weak intensity claudin-4 staining in a borderline MCN (score 1). (F) Strong intensity claudin-4 staining in an MCN carcinoma (score 2). (G) Negative claudin-18 staining in an MCN adenoma (score 0). (H) Weak intensity claudin-18 staining in a borderline MCN (score 1), (I) Strong intensity claudin-18 staining in an MCN carcinoma (score 2) (A-I, x400). 


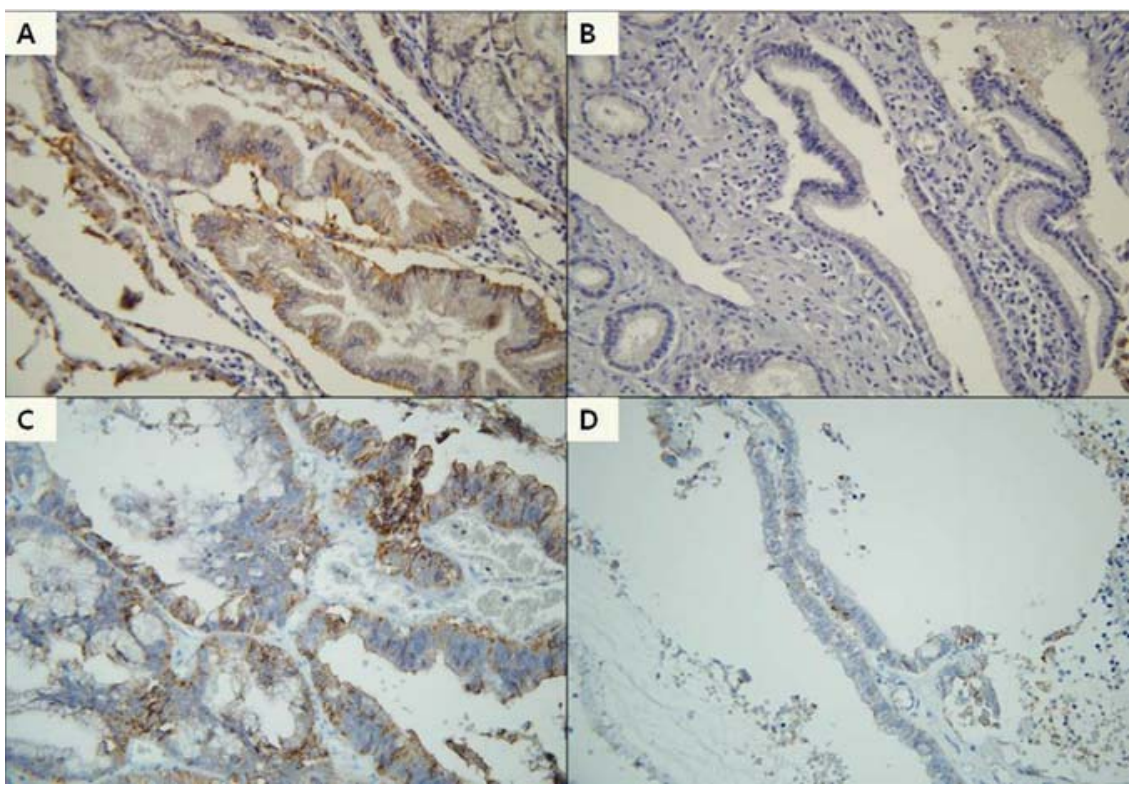

Figure 4. Claudin-4 and -18 expression in differing grade of dysplasia in the same section. (A and B) In the same section of IPMN carcinoma, while carcinoma cells are strongly positive, cells at benign lesion are negative for claudin-18 expression. (C and D) In the same section of MCN carcinoma, while carcinoma cells show robust expression of clauding-4, cells at borderline lesion show faint expression. (A-D, x400).

A

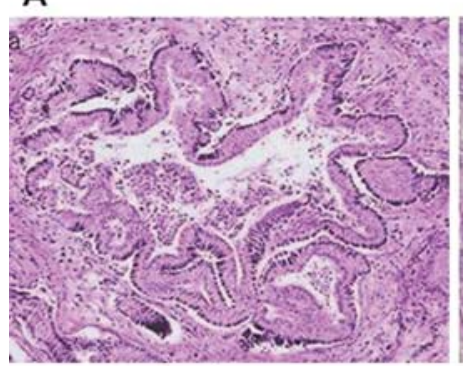

Gastric type

B

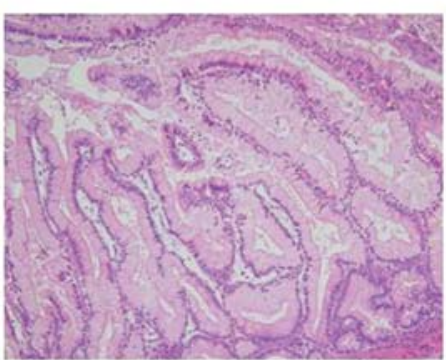

Intestinal type

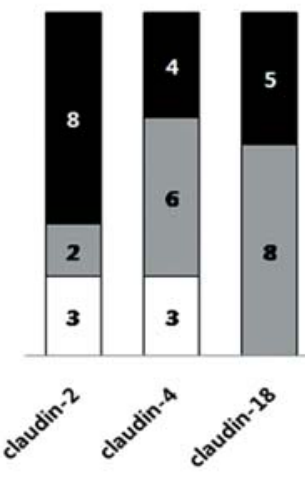

Intestinal type

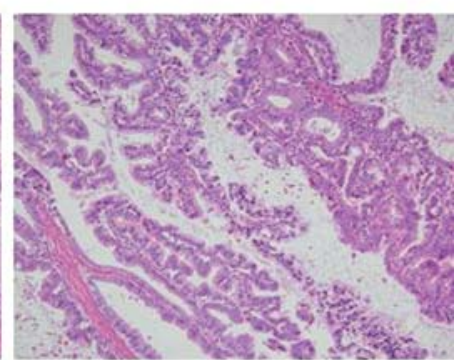

Pancreato-biliary type
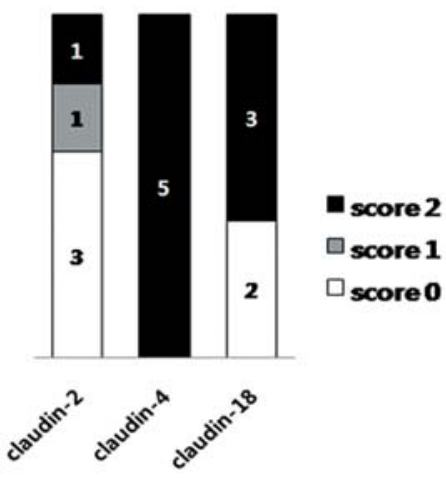

Pancreato-biliary type

Figure 5. (A) Histological IPMN subtypes by hematoxylin and eosin staining. Gastric type IPMN mimics gastric foveolae and has thick finger-like papillae and abundant flare areas. Intestinal type IPMN mimics an intestinal villous neoplasm and has villous papillae. Pancreatobiliary type IPMN mimics a cholangiopapillary neoplasm and has thin branching complex papillae with moderate amphophilic cytoplasm (x400). (B) Claudin-2, -4 , and -18 expression relative to IPMN histological subtype quantitative score. Claudin-2 expression was stronger in intestinal type IPMN, although this was not statistically significant ( $\mathrm{p}=0.374)$. Claudin-4 expression was significantly higher in pancreato-biliary type IPMN $(100 \%)$; $(\mathrm{p}=0.015)$. Claudin-18 expression was stronger in gastric type IPMN ( $\mathrm{p}=0.023)$.

while more differentiated area showed weak or negative expression (Fig. 4).

Histological subtypes of IPMN were categorized into gastric, intestinal, pancreato-biliary, or oncocytic (Fig. 5A).
There were no cases of oncocytic IPMN in this study. Claudin-2 expression was stronger in intestinal type IPMN, although this was not statistically significant $(\mathrm{p}=0.374)$. The grade of claudin-4 expression was significantly higher in pancreato- 
biliary type IPMN (100\%) $(\mathrm{p}=0.015)$, whereas claudin-18 expression was stronger in gastric type IPMN $(\mathrm{p}=0.023)$ (Fig. 5B).

\section{Discussion}

In the present study, we investigated the expression pattern of tight junction proteins, claudin-2, -4 , and -18 through immunohistochemical staining in surgically resected tissues of pancreatic mucinous tumors. Mucin-producing pancreatic tumors represented by IPMN and MCN are premalignant conditions and occasionally evolve into invasive carcinoma (3). Although IPMN and MCN are histologically very similar to each other, these are definitely separate entities differing in gender predominance, age prevalence, and location of tumor within the pancreas $(22,23)$.

IPMN is characterized by cystic dilatation of pancreatic ducts in which an intraductal proliferation of neoplastic mucinproducing cells is usually arranged in papillary patterns (24). Although IPMNs are estimated to account for 5\% of pancreatic neoplasms, they are not infrequently found on pancreatic imaging such as ultrasonography, computed tomography, and magnetic resonance imaging and thought to be more common than previously recognized (25). MCN is presumably a de novo cystic tumor which gives rise to cystic dilatation of native ducts and characterized by an ovarian type of stroma. Although IPMN and MCN have some features that overlap, they can be distinguished by clinical, gross, and microscopic findings. While IPMN is more common in elderly male and often localizes in the head of pancreas, MCN occurs predominantly in perimenopausal female and localizes in the tail of pancreas $(22,23)$. Whereas mucin-filled cysts of IPMN communicate with pancreatic ducts, MCN cysts typically do not communicate with the ductal system and often have thick walls. The presence of ovarian stroma is diagnostic for MCN and has almost become a requirement for the diagnosis of this tumor type (26). Occasionally, it is difficult to differentiate both conditions solely based on histology, especially when ovarian type stroma is not seen in a suspected MCN case. Molecular features might be of help differentiating the diseases (2), however, there have been only a few reports and none of them have dealt with claudin proteins.

We aimed to find different features between IPMN and MCN at molecular level. In this study, however, both IPMN and MCN revealed similar patterns of claudin expression. Though majority of the mucinous tumors were reactive to claudin immunostaining, there were obvious differences of expression pattern according to the type of claudin proteins. While advanced histological lesions showed more robust expression of claudin- 4 and -18 , claudin-2 was predominantly expressed in benign adenomas. These findings were again supported by the findings that some cases having both borderline malignant and carcinoma lesions in the same section, tumor cells at carcinoma were positive for claudin-4 and -18 , while tumor cells at borderline lesion showed weak or negative expression.

Claudins are essential components of tight junction that are critical for the maintenance of cellular integrity and polarity. Based on the role of claudins in the formation of tight junction, it has been hypothesized that the loss of claudin expression may reduce cell adhesion and thereby increase tumor cell motility and invasive potential (27). Paradoxically, not only down-regulation but also up-regulation of claudins in many different cancers have been reported (14-21). Overexpression may allow the tumor cells to overcome the loss of cell adhesion resulting from the loss of other adhesion molecules (28). Alternatively, up-regulation and/or aberrant expression of claudins may directly interfere with tight junction formation and function, thereby contributing to neoplastic transformation (29). Up-regulation of claudins may also affect cell signaling pathways through their interactions with ZO-1, via binding domains at the carboxyl terminus. ZO-1 interacts with several signaling proteins associated with the neoplastic process, such as the Ras substrate AF- $6, \mathrm{G}$ proteins, and connexin 43 (30). In a study using human ovarian surface epithelial cells that constitutively express wild-type claudin-3 and -4 (31), the overexpression of claudin-3 and -4 did not result in functional loss of tight junctions and they promoted invasiveness.

The high specificity of claudin expression in cancer may represent useful molecular markers for various cancers (8). For example, a set of four markers, including claudin-3, was found to be sufficient to accurately identify all 158 ovarian cancers tested, including eight early-stage serous cancers (32). In addition, claudin expression may be used as a prognostic indicator, like low claudin-1 expression is associated with a poor prognosis in stage II colon cancer (15). Claudin-2 has been shown to be up-regulated in colorectal cancer (33) and closely correlated to gastric carcinogenesis (34). Claudin-10 expression is also an independent prognostic factor for hepatocellular carcinoma recurrence after curative hepatectomy (35). In particular, a series of previous studies demonstrated claudin- 4 expression in pancreatic cancer and IPMN $(18,20,36)$. Moreover, targeting claudin-4 expressing tumors with Clostridium perfringens enterotoxin represented a promising new treatment modality for pancreatic cancer, and the data showed the feasibility of identifying new cancer treatment targets using highthroughput expression profiling analyses $(17,37)$. In another study, claudin-18 was highly overexpressed in pancreatic infiltrating ductal adenocarcinoma as compared with the normal reactive ducts (37). Claudin-18 was also up-regulated in gastric carcinoma (38).

Although a number of studies demonstrated the implication of claudins in tumorigenesis including claudin-4 overexpression in IPMN, the expression of claudin- 2 and -18 in IPMN and MCN has not been addressed. Among the 24 types of claudins, claudin-2 is unique in that its expression causes tight junctions to become leaky (39), whereas other claudins seal up tight junctions. An earlier study showed that increased expression of claudin-2 in Madin-Darby canine kidney cells increased the leakiness of TJs (40). These findings may explain the reciprocal changes of claudin-2 vs. claudin- 4 and -18 expressions in pancreatic mucinous tumors in our study.

In this study, we also addressed the relationships between the histological subtypes of IPMN and claudin expression. The gastric type commonly forms branched duct-type IPMN with low-grade atypia thus harboring low malignant potential. Others more often present with marked atypia, mostly 
corresponding to borderline category or carcinoma, and are more aggressive $(22,41,42)$. In our study, 2 of $11(18.2 \%)$ gastric types were adenomas and 4 of $5(80 \%)$ pancreatobiliary types were carcinomas. Because the number of each histological subtype was too small and the oncocytic type was absent, these data did not represent the exact expression pattern of claudins according to sybtype of IPMN. As shown in Fig. 5, however, claudin-2 was more often expressed in gastric $(81.8 \%)$ and intestinal types $(76.9 \%)$ compared to pancreato-biliary types $(40 \%)(\mathrm{p}=0.374)$, which suggests claudin-2 may be a marker of benign mucinous tumors of pancreas. Contrary to claudin-2, claudin-4 revealed robust expression in pancreato-biliary types $(p=0.015)$. These findings suggest that the pattern of claudin expression can be useful in differentiating histological subtypes of IPMN.

In summary, our study suggests that pancreatic mucinous tumors, IPMN and MCN, frequently express claudin-2, -4 and -18 proteins. As the tumors advance, the expression level of claudin- 4 and -18 increases, whereas claudin- 2 decreases. Though further clarification needs to solve the role of claudins, this study suggests that claudins are involved in the tumorigenesis of pancreatic mucinous tumors and may be useful in differentiating histological grades and subtypes.

\section{Acknowledgements}

This study was supported by the Korean Research Foundation Grant funded by the Korea Government (R012007-000-20710-0).

\section{References}

1. Kosmahl M, Pauser U, Peters K, et al: Cystic neoplasms of the pancreas and tumor-like lesions with cystic features: a review of 418 cases and a classification proposal. Virchows Arch 445: 168-178, 2004

2. Sawai H, Okada Y, Funahashi H, Matsuo Y, Tanaka M and Manabe T: Immunohistochemical analysis of molecular biological factors in intraductal papillary-mucinous tumors and mucinous cystic tumors of the pancreas. Scand J Gastroenterol 39: 1159-1165, 2004.

3. Schmidt CM, White PB, Waters JA, et al: Intraductal papillary mucinous neoplasms: predictors of malignant and invasive pathology. Ann Surg 246: 644-651, 2007.

4. Adsay NV, Merati K, Andea A, et al: The dichotomy in the preinvasive neoplasia to invasive carcinoma sequence in the pancreas: differential expression of MUC1 and MUC2 supports the existence of two separate pathways of carcinogenesis. Mod Pathol 15: 1087-1095, 2002

5. Hruban RH, Takaori K, Klimstra DS, et al: An illustrated consensus on the classification of pancreatic intraepithelial neoplasia and intraductal papillary mucinous neoplasms. Am J Surg Pathol 28: 977-987, 2004

6. Goh BK, Tan YM, Cheow PC, et al: Cystic neoplasms of the pancreas with mucin-production. Eur J Surg Oncol 31: 282-287, 2005.

7. Suzuki Y, Atomi Y, Sugiyama M, et al: Cystic neoplasm of the pancreas: a Japanese multiinstitutional study of intraductal papillary mucinous tumor and mucinous cystic tumor. Pancreas 28: 241-246, 2004.

8. Morin PJ: Claudin proteins in human cancer: promising new targets for diagnosis and therapy. Cancer Res 65: 9603-9606, 2005 .

9. Schneeberger EE and Lynch RD: The tight junction: a multifunctional complex. Am J Physiol Cell Physiol 286: C1213-C1228, 2004.

10. Tsukita S, Furuse M and Itoh M: Multifunctional strands in tight junctions. Nat Rev Mol Cell Biol 2: 285-293, 2001

11. Mitic LL and Anderson JM: Molecular architecture of tight junctions. Annu Rev Physiol 60: 121-142, 1998.
12. Ren J, Hamada J, Takeichi N, Fujikawa S and Kobayashi H: Ultrastructural differences in junctional intercellular communication between highly and weakly metastatic clones derived from rat mammary carcinoma. Cancer Res 50: 358-362, 1990.

13. Martin TA and Jiang WG: Tight junctions and their role in cancer metastasis. Histol Histopathol 16: 1183-1195, 2001.

14. Tokes AM, Kulka J, Paku S, et al: Claudin-1, -3 and -4 proteins and mRNA expression in benign and malignant breast lesions: a research study. Breast Cancer Res 7: R296-R305, 2005.

15. Resnick MB, Konkin T, Routhier J, Sabo E and Pricolo VE: Claudin-1 is a strong prognostic indicator in stage II colonic cancer: a tissue microarray study. Mod Pathol 18: 511-518, 2005.

16. Gress TM, Muller-Pillasch F, Geng M, et al: A pancreatic cancer-specific expression profile. Oncogene 13: 1819-1830, 1996.

17. Michl P, Buchholz M, Rolke M, et al: Claudin-4: a new target for pancreatic cancer treatment using Clostridium perfringens enterotoxin. Gastroenterology 121: 678-684, 2001.

18. Nichols LS, Ashfaq R and Iacobuzio-Donahue CA: Claudin 4 protein expression in primary and metastatic pancreatic cancer: support for use as a therapeutic target. Am J Clin Pathol 121: 226-230, 2004.

19. Hough CD, Sherman-Baust CA, Pizer ES, et al: Large-scale serial analysis of gene expression reveals genes differentially expressed in ovarian cancer. Cancer Res 60: 6281-6287, 2000.

20. Sato N, Fukushima N, Maitra A, et al: Gene expression profiling identifies genes associated with invasive intraductal papillary mucinous neoplasms of the pancreas. Am J Pathol 164: 903-914, 2004.

21. Rangel LB, Agarwal R, D'Souza T, et al: Tight junction proteins claudin-3 and claudin- 4 are frequently overexpressed in ovarian cancer but not in ovarian cystadenomas. Clin Cancer Res 9: 2567-2575, 2003

22. Yamaguchi K, Yokohata K, Noshiro H, Chijiiwa K and Tanaka M: Mucinous cystic neoplasm of the pancreas or intraductal papillary-mucinous tumour of the pancreas. Eur J Surg 166: $141-148,2000$

23. Volkan Adsay N: Cystic lesions of the pancreas. Mod Pathol 20 (Suppl. 1): S71-S93, 2007.

24. Furukawa T, Kloppel G, Volkan Adsay N, et al: Classification of types of intraductal papillary-mucinous neoplasm of the pancreas: a consensus study. Virchows Arch 447: 794-799, 2005.

25. Adsay NV: The 'new kid on the block': intraductal papillary mucinous neoplasms of the pancreas: current concepts and controversies. Surgery 133: 459-463, 2003.

26. Tanaka M, Chari S, Adsay V, et al: International consensus guidelines for management of intraductal papillary mucinous neoplasms and mucinous cystic neoplasms of the pancreas. Pancreatology 6: 17-32, 2006.

27. van Grevenstein WM, Hofland LJ, Jeekel J and van Eijck CH: The expression of adhesion molecules and the influence of inflammatory cytokines on the adhesion of human pancreatic carcinoma cells to mesothelial monolayers. Pancreas 32: 396-402, 2006

28. Heinzelmann-Schwarz VA, Gardiner-Garden M, Henshall SM, et al: Overexpression of the cell adhesion molecules DDR1, Claudin 3, and Ep-CAM in metaplastic ovarian epithelium and ovarian cancer. Clin Cancer Res 10: 4427-4436, 2004

29. Resnick MB, Gavilanez M, Newton E, et al: Claudin expression in gastric adenocarcinomas: a tissue microarray study with prognostic correlation. Hum Pathol 36: 886-892, 2005.

30. Itoh M, Furuse M, Morita K, Kubota K, Saitou M and Tsukita S: Direct binding of three tight junction-associated MAGUKs, ZO-1, ZO-2, and ZO-3, with the $\mathrm{COOH}$ termini of claudins. J Cell Biol 147: 1351-1363, 1999.

31. Agarwal R, D'Souza T and Morin PJ: Claudin-3 and claudin-4 expression in ovarian epithelial cells enhances invasion and is associated with increased matrix metalloproteinase-2 activity. Cancer Res 65: 7378-7385, 2005.

32. Lu KH, Patterson AP, Wang L, et al: Selection of potential markers for epithelial ovarian cancer with gene expression arrays and recursive descent partition analysis. Clin Cancer Res 10: 3291-3300, 2004

33. Kinugasa T, Huo Q, Higashi D, et al: Selective up-regulation of claudin- 1 and claudin-2 in colorectal cancer. Anticancer Res 27: 3729-3734, 2007. 
34. Song X, Li X, Tang Y, et al: Expression of claudin-2 in the multistage process of gastric carcinogenesis. Histol Histopathol 23: 673-682, 2008

35. Cheung ST, Leung KL, Ip YC, et al: Claudin-10 expression level is associated with recurrence of primary hepatocellular carcinoma. Clin Cancer Res 11: 551-556, 2005.

36. Tsukahara M, Nagai H, Kamiakito T, et al: Distinct expression patterns of claudin-1 and claudin-4 in intraductal papillarymucinous tumors of the pancreas. Pathol Int 55: 63-69, 2005.

37. Karanjawala ZE, Illei PB, Ashfaq R, et al: New markers of pancreatic cancer identified through differential gene expression analyses: claudin 18 and annexin A8. Am J Surg Pathol 32: 188-196, 2008

38. Hewitt KJ, Agarwal R and Morin PJ: The claudin gene family: expression in normal and neoplastic tissues. BMC Cancer 6: $186,2006$.
39. Amasheh S, Meiri N, Gitter AH, et al: Claudin-2 expression induces cation-selective channels in tight junctions of epithelial cells. J Cell Sci 115: 4969-4976, 2002.

40. Furuse M, Furuse K, Sasaki H and Tsukita S: Conversion of zonulae occludentes from tight to leaky strand type by introducing claudin-2 into Madin-Darby canine kidney I cells. J Cell Biol 153: 263-272, 2001.

41. Ishida M, Egawa S, Aoki T, et al: Characteristic clinicopathological features of the types of intraductal papillary-mucinous neoplasms of the pancreas. Pancreas 35: 348-352, 2007.

42. Ban S, Naitoh Y, Mino-Kenudson M, et al: Intraductal papillary mucinous neoplasm (IPMN) of the pancreas: its histopathologic difference between 2 major types. Am J Surg Pathol 30: 1561-1569, 2006. 